\title{
Analytical challenges in the qualification and validation of pharmacodynamic biomarkers
}

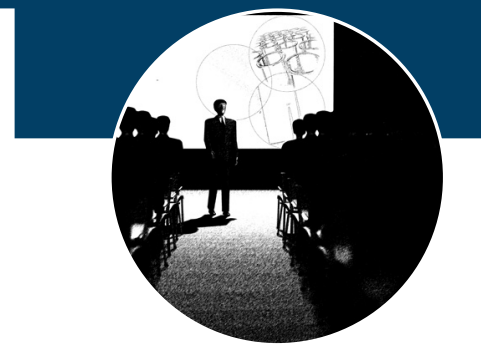

The Royal Society of Chemistry

Burlington House, London, UK, 15 March 2011

This I-day workshop, held in association with the Royal Society of Chemistry Analytical Biosciences Group, discussed current concepts in the qualification and validation of biomarker assays for the measurement of pharmacodynamic responses to drugs and vaccines. The venue was Burlington House, the prestigious home of the Royal Society of Chemistry, with delegates drawn from academia, pharmaceutical companies and CROs.

Keywords: biomarker $₫$ conference $₫$ method qualification $\approx$ method validation $\approx$ pharmacodynamic $₫ \mathrm{PK} / \mathrm{PD}$

The workshop was chaired by Ken Leiper (Joint Pharmaceutical Analysis Group) who welcomed delegates and introduced the speakers, who covered a wide range of topics. In addition to standard ELISA formats, the meeting illustrated the application of other platform technologies including flow cytometry, enzyme-linked immunospot (ELISpot), quantitative PCR (Q-PCR), Gyros, LC-MS/MS and multiplexed platforms including Luminex and Meso Scale Discovery ${ }^{\circledR}$ (MSD). The concept of a 'fit-forpurpose' method qualification and validation was discussed along with the selection of suitable reference materials, quality control (QCs) and relevant acceptance criteria.

\section{Biomarkers \& biomeasures: key enablers for translational} pharmacokinetics/pharmacodynamics in drug discovery \& development

The opening keynote lecture by Piet Van Der Graaf (Pfizer) discussed biomarkers and biomeasures, considered to be key enablers for translational pharmacokinetics/pharmacodynamics (PK/PD) in drug discovery and development [1]. Preclinical or translational PK/PD modeling and simulation $(M \& S)$ is a relatively new area in drug discovery and until recently was mainly restricted to academic research [2]. However, it is increasingly being recognized that successful implementation of PK/PD reasoning in early drug discovery could have an even greater impact on the overall efficiency and success of pharmaceutical research as comparable investments in late-stage M\&S. This is because arguably the most significant challenge facing the pharmaceutical industry is compound attrition resulting from the failure of preclinical efficacy and safety model data to translate into human proof-of-mechanism/concept studies. Therefore, it has been suggested that PK/PD M\&S can also play a significant role in early preclinical drug discovery and can provide a framework for translational research that links, in a quantitative manner, the interactions between a drug (or combination of drugs), pharmacological targets, physiological pathways and, ultimately, integrated disease systems.

Not surprisingly, with increased interest in its relevance to preclinical research, PK/PD has evolved towards a more mechanistic approach, and (semi-)mechanistic PK/PD models are now advocated not only by academic and industrial researchers, but also by regulators. However, the development of mechanism-based methods for cross-species scaling of PD parameters is still in its infancy, although some recent examples have suggested that allometric scaling may be applicable to predict not only PK but also PD responses in humans from data obtained in preclinical in vitro and in vivo models. A recent development in this area is based on the growing realization that innovation could be dramatically catalyzed by creating synergy at the interface between systems biology and PK/PD, two disciplines that, until now, have largely existed in 'parallel universes' with a limited track record of impactful collaboration. This has led to the emergence of systems pharmacology. Broadly speaking, this is the quantitative analysis of the
Richard Houghton ${ }^{\dagger 1}$ \& Joseph Chamberlain ${ }^{2}$

'Quotient Bioresearch Ltd, Newmarket Road, Fordham, Cambridge, CB7 5WW, UK ${ }^{2}$ Joint Pharmaceutical Analysis Group, Royal Society of Chemistry ${ }^{\dagger}$ Author for correspondence:

Tel.: +44163872 4216 Fax: +44 1638720500

E-mail: richard.houghton@ quotientbioresearch.com 
dynamic interactions between $\operatorname{drug}(s)$ and a biological system. In other words, systems pharmacology aims to understand the behavior of the system as a whole, as opposed to the behavior of its individual constituents; thus it has become the interface between PK/PD and systems biology. It applies the concepts of systems engineering, systems biology and PK/PD to the study of complex biological systems through iteration between computational and/or mathematical modeling and experimentation. Biomeasures (i.e., quantitative information about system properties such as target expression levels and turnover dynamics) are key enablers for success in systems pharmacology in a similar way as biomarkers have been for $\mathrm{PK} / \mathrm{PD}$ and more investments are required in this area.

Piet Van Der Graaf concluded by saying, "M\&S in its broadest sense will only achieve its full potential in tackling $R \& D$ attrition when the different components and disciplines operating at different stages of the R\&D cycle are fully integrated into an 'enhanced quantitative drug discovery and development' (EQD3) paradigm”.

\section{Adaption \& validation of commercial ligand-binding assay kits}

John Allinson (Icon) reviewed the various considerations required when validating commercially available ligand-binding assay kits for use in biomarker quantification [3]. Whilst commercially available kits are readily available, they may have a limited standard curve or material, the lower limit of detection may not be the LLOQ, sample stability may not have been extensively evaluated, the disease state is generally not evaluated, they can be expensive, with unknown lotto-lot variability and may not be optimized for your intended use. It is important, therefore, to ensure the kit undergoes 'fit-for-purpose' method validation as out-of-the-box performance cannot be assumed. One definite consideration is the authenticity of reference materials; an alternative source of material should be sought in order to prepare QC samples.

The dangers of inappropriate use of commercial kits were illustrated with a number of illuminating examples. Allinson also presented a wealth of data generated on a number of commercial platforms demonstrating that there is no 'one-size-fits-all' technology, but rather a number of platforms are required in laboratories performing biomarker work. He also looked at the importance of demonstrating parallelism of a method. He concluded by saying that commercial assays must be interrogated appropriately, a laboratory should have a mix of suitably qualified scientific staff that can interpret the data correctly with an importance to share relevant data across different platforms.

\section{Small-molecule LC-MS/MS}

Biomarkers are not limited to large molecules such as proteins, as demonstrated by Richard Houghton (Quotient Bioresearch) in a presentation on quantification of small-molecule steroid biomarkers using LC-MS/MS. A multiplexed high-throughput LC-MS/MS method for the measurement of seven steroids in human urine was developed. The method was subject to the rigors of a regulated validation using a generic surrogate matrix calibration strategy, used routinely at Quotient for the quantification of endogenous analytes [4]. The multiplexed assay was used to good advantage enabling the measurement of steroid concentrations and calculation of a steroid ratio indicative of inhibition of the $11 \beta$-hydroxy steroid dehydrogenase enzyme from a single LC-MS/MS run. Data generated using this method in support of two Phase I clinical studies, with two different drugs, illustrated the power of PD data in demonstrating proof-of-principle, potency, drug kinetics and food effects at this relatively early stage in the development process. Error analysis of QC samples suggested that batch acceptance criteria would be better applied to the end point ratio rather than absolute concentrations of analytes.

\section{Transcriptomic Q-PCR assays}

The study of transcriptomics, also referred to as expression profiling, examines the expression level of mRNAs in a given cell population, often using high-throughput techniques based on DNA microarray technology. Chaminda Salgado (NDA Analytics) described the validation of transcriptomic Q-PCR assays for personalized medicine in a detailed and wideranging account of this complex subject. $\mathrm{He}$ covered topics such as the rationale for real-time PCR, gene expression, current uses and issues including quantitative range, robustness, speed, throughput, portability and proof-of-concept. Examples were given of current PCR diagnostics, but Salgado warned, "beware of advisory literature surrounding Q-PCR." There are many interdependencies from assay design to chemistry and reverse transcriptase choices. It is unfeasible to test every permutation for every step of the process especially relating to one's own targets of interest; however, over-reliance on vendor software should also be avoided. 
Nevertheless, there have been many successes in diagnostic therapy for individual patients, pathogen diagnostics and mutation detection, concluded Salgado.

\section{Flow cytometry for measuring the PD of theurapeutic antibodies}

Flow cytometry is a mature technology (at least 40 years old), which has been used for diagnostic applications (leukaemia, HIV and transplantation), said Kevin Maskell (Merck Millipore). It is versatile (high-throughput, cell sorting and multiparameter) and can be adapted successfully for PD biomarker analysis (immune subsets, protein expression). Maskell described the technique for measuring the PD of therapeutic antibodies, emphasising the importance of practical sample collection procedures tailored to suit analysis of clinical samples. He suggested that great attention should be paid to the qualification of reagents, not relying on the claims of the supplier. For validation, most assays conform to a 'fit-for-purpose' validation, but can be more quantitative if calibration and QC materials are available. A critical question is whether PD data can be used as PK data. Flow cytometry can be used for PK using antigen-coated beads or stably expressing cell lines to bind drug in the patient's serum. The bound drug is then quantified using an anti-idiotype antibody, but usually traditional methods are more appropriate as they are more precise and sensitive. However if the drug targets immune cells, and low doses are administered to a subject or the drug is delivered subcutaneously, it can be difficult to detect any drug using traditional methods in the serum. The use of receptor occupancy (saturation) flow cytometry analysis is more approapriate. This should be done using a noncompetitive detection reagent for the drug target or by characterizing the drug-fluorochrome and antidrug antibodies for a number of antibody molecules. Flow cytometry for PK can prove to be more variable than traditional methods.

\section{Cytokine release assays using multiplexed technologies}

Hypercytokinemia is a potentially fatal immune reaction consisting of a positive feedback loop between cytokines and immune cells, with highly elevated levels of various cytokines said Christopher Kirton (Huntingdon Life Sciences). It is essential to monitor cytokine release in clinical trials where there is potential for an immune response. Kirton first established the predictive value of such assays and then described ex vivo bioassays to detect cytokine release by multiplex technologies, comparing cytometric bead array and the proprietary technology of MSD, which provides solutions for multiplex biological assays, including assays for biomarkers, cytokines and phosphoproteins. MSD's platform is based on their Multi-Array ${ }^{\circledR}$ technology, a proprietary combination of patterned arrays and electrochemiluminescence detection, enabling large numbers of measurements with exceptional sensitivity, wide dynamic range and convenience. Whilst both platforms offer multiplex technology they differ in their application to the drug-development process. MSD offers a sensitive high-throughput platform that is appropriate for early-stage screening assays and clinical support, whereas cytometric bead array technology has a lower throughput but uses more available technology, which makes it useful for assay development and late-stage work. Ultimately, it depends on the intended application - the right tool for the right job, concluded Kirton.

\section{ELISpot for the determination of}

\section{vaccine-specific immune responses}

James Francis (Immune Targeting Systems) gave a brief introduction to the company, focusing on the basic principles of ELISpot. Most ELISpot techniques are based on the sandwich ELISA principle, but can be 200 -fold more sensitive than ELISA. It is often used to measure antigenspecific responses in the fields of vaccine research, AIDS research, cancer research, monitoring of infectious diseases, and allergy and transplantation research. Francis described the validation of the ELISpot assay to test clinical samples and the utility of ELISpot assays from preclinical to clinical studies. Validation procedures must identify variable aspects of the assay that may create problems, such as the choice of reagents, the concentration of coating and detection antibodies, the number of cells to use in the assay, and the choice of suitable positive and negative controls.

ELISpot has found utility in vaccine research in obtaining preclinical immunogenicity data (mouse IFN- $\gamma$, rat IFN- $\gamma$, ferret IFN- $\gamma$, human IFN- $\gamma$, IL-2, granzyme and perforin), in toxicology (rat IFN- $\gamma$ ) and in Phase I clinical studies (human IFN- $\gamma$ ). An interesting variation was the use of different colored spots in the dual color ELISpot assay, for example to measure IL-2 and IL-4. Francis concluded that ELISpot was suitable for functional analysis of immune cells with very high sensitivity and the potential for high throughput. It was simple to conduct with relatively low start-up costs and provided a robust tool for use in vaccine immunogenicity studies. 
